\title{
Novel Vestigial Sideband Modulation Scheme to Enhance the SNR in Radio Over Fiber Systems
}

\author{
Hum Nath PARAJULI, Eszter UDVARY \\ Dept. of Broadband Info-Communication and Electromagnetic Theory, Budapest University of Technology and \\ Economics, 3 Muegyetem rakpart, 1111 Budapest, Hungary \\ \{hum.nath.parajuli, udvary\}@hvt.bme.hu \\ Submitted February 8, 2017 / Accepted July 16, 2017
}

\begin{abstract}
The optical single sideband (OSSB) scheme is a common choice in radio over fiber system for compensating chromatic dispersion (CD) but it suffers from low signal to noise ratio (SNR). We present the novel technique for SNR improvement using unequal sideband intensity based modulation scheme, we name it optical vestigial sideband modulation scheme (OVSB). To analyze such scheme we use the series Mach-Zehnder modulator (MZM) and phase modulator (PM) configuration. In this configuration by operating MZM at quadrature point and properly tuning the PM index the individual intensity of the first order sidebands and higher order harmonics can be controlled independent to each other. The optimum operating point of PM for maximizing the SNR can be identified by reducing the harmonic distortion and increasing the first order sidebands power. We experimentally validated the proposed OVSB scheme generation method and provide its theoretical proof. We evaluate the symbol error rate (SER) performance using 4QAM signal for OSSB and OVSB and show the improved performance of OVSB scheme compared to OSSB scheme. We also discuss the harmonic distortion behavior of the series MZM and PM configuration.
\end{abstract}

\section{Keywords}

Optical-wireless, optical single sideband, optical vestigial sideband, radio over fiber, signal to noise ratio

\section{Introduction}

In radio over fiber (RoF) system a baseband signal upconverted with radio frequency (RF) carrier modulates the optical carrier and is transmitted through a low loss and huge bandwidth optical fiber. RoF links have the ability to deliver RF signals into remote cells without significant degradation of the transmitted qualities such as RF, modulation formats etc. RoF technology allows small cell deployment in the remote area offering Gbps wireless access and makes the network simple and scalable. The $5 \mathrm{G}$ deployment goal by 2020 is to provide $1-10$ Gbps wireless signal for the end user [1], [2]. To achieve the 5G deploy- ment goal, realization of high signal to noise ratio (SNR) of the received signal is one of the important issues.

During the process of electrical to optical conversion (E-O), the optical carrier is intensity modulated and optical sidebands are created. Optical double sideband (ODSB), optical single sideband (OSSB) and optical carrier suppressed double sideband (OCS-DSB) schemes are the major sideband modulation schemes in RoF technology. The choice of these sideband modulation schemes for any RoF system depends on the capacity and minimum reach that RoF system requires to fulfill. When the ODSB modulated $\mathrm{RF}$ signal is transmitted via optical fiber, each sidebands travel at different velocities. So, different sidebands will arrive at the receiver at different times thereby changing the phase relationship between sidebands. If the sidebands experience a total phase difference of $180^{\circ}$ relative to the optical carrier the RF signal will vanish [3], [4]. Depending on the amount of phase shifts due to chromatic dispersion (CD), cyclic power fading of the detected RF signal occurs. Thus, the ODSB scheme is not suitable for a high frequency and long reach RoF system; if the constant received signal power is important for wide range of frequencies. OSSB and OCS-DSB are the preferred schemes in terms of compensating CD. The OCS-DSB scheme cannot be used for vector signal transmission because it doubles signal frequency, phase and phase noise at the receiver. As a consequence the transmitted signal will appear modified as well as distorted when detected by the receiver [5]. OSSB scheme can deliver the vector signal without modification of the sent signal however suffers from serious SNR degradation due to the strong dc component present in the detected signal.

To maximize the SNR of the OSSB based RoF link, the optimization of optical carrier to sideband ratio (OCSR) at $0 \mathrm{~dB}$ is important [6], [7]. Generally, to generate the OSSB scheme either an electro optic modulator along with an optical filter, or a dual electrode modulator (DMZM) is used [8], [9]. With optical filter method to maximize SNR, the optical carrier power has to be attenuated using tunable optical filter to make it equal to sideband power. Similarly, to achieve OCSR $=0 \mathrm{~dB}$ in DMZM method it is required to change the bias voltage of the modulator. The DMZM consists of the MZM and PM in parallel [10]. By changing 
the bias voltage of DMZM for OCSR $=0 \mathrm{~dB}$ operation, the internal MZM goes in nonlinear region of MZM transfer curve, ultimately reducing the SNR. Additionally, optical band pass filter is required to suppress undesired harmonics generated from the nonlinear region operation. In the DMZM method, if the MZM is operated in linear region, to make optical carrier power and sideband power equal for OCSR $=0 \mathrm{~dB}$ condition, additional tunable optical filter is required to suppress the optical carrier. Thus, optical amplifier is also required to enhance the optical power. Another approach of operating at OCSR $=0 \mathrm{~dB}$ has been demonstrated in [11] using a strong optical injection locked laser. This method requires two lasers and precise measurements.

By considering all the above mentioned complexities to enhance the SNR in the RoF systems, we propose to use the unequal sideband intensity based scheme, we name it vestigial sideband modulation scheme (OVSB). To analyze this scheme, we use the series MZM and PM configuration based sideband generation method [12]. In this method the SNR can be optimized by properly controlling the intensities of the first order sidebands named as upper sideband (USB) and lower order sideband (USB) and higher order harmonics. The signal power can be increased through the use of partially suppressed first order sidebands and the harmonic distortion can be reduced by reducing the harmonics' power. These effects can be realized by properly tuning the PM index in series MZM and PM configuration. The choice of the proper modulation parameters can cancel the CD effect as well as enhances the SNR.

This paper is organized as follows. In Sec. 1.1 we will show the generation method of the OVSB and OSSB scheme using the series MZM and PM configuration. Also, we describe how OVSB method is advantageous to optimize the SNR of the link in comparison to the OCSR = $0 \mathrm{~dB}$ method of [6], [11]. Section 2 gives the clear analytical description on the OSSB and OVSB generation method using the series MZM and PM configuration. This section also describes the major transmission impairments associated with RoF systems. Section 3 provides the experimental validation of the proposed OVSB generation method. Section 4 presents by numerical simulation the improved performance of OVSB scheme compared to OSSB scheme for series MZM and PM configuration. Section 5 concludes the paper.

\subsection{Proposed Method for Generation of OVSB Scheme}

The proposed method of generating OVSB scheme using series MZM and PM configuration is shown in Fig. 1.

Since the proposed configuration does not require an optical tunable filter, the problems of a filter method like filter instability and precise frequency tuning are avoided. Also, PM does not require a bias voltage, so it is less affected by environmental fluctuations. With the proposed series configuration of MZM and PM, it allows to choose the MZM and PM parameters independent to each

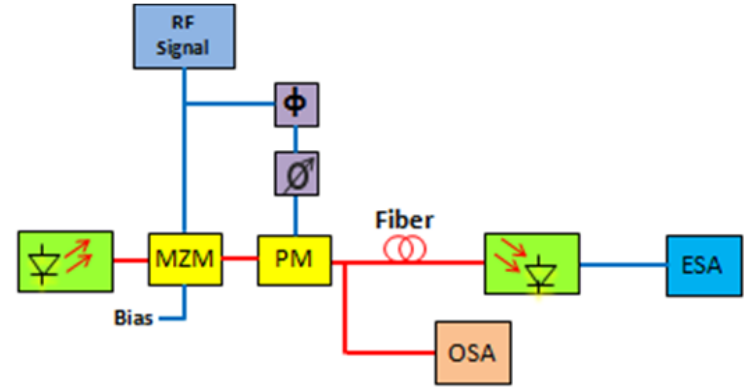

Fig. 1. The functional block diagram for generation and performance analysis of the proposed OVSB scheme with OSSB scheme. OSA: optical spectrum analyzer, ESA: electrical signal analyzer.

other. In the proposed scheme, the MZM can be operated in a linear region of the MZM transfer curve and only PM index can be tuned. By tuning the PM index, each first order sideband's intensities (USB and LSB) can be controlled independently and OVSB scheme can be generated. The maximization of the SNR can be done by employing unequal sideband intensities in which one of the sideband is not suppressed completely to enhance the signal power unlike in the case of OSSB. The undesired higher order sideband intensity can also be controlled properly to minimize the harmonic distortion. The required PM index for maximum SNR depends on the used frequency and fiber length. The SNR depends not only on the received signal power but also on the harmonic distortion of the RoF system. In this way, the OVSB scheme enhances the strength of the received signal as well as balances the intensities of the generated harmonic components to minimize the distortion, which improves the system performance compared to OSSB scheme. Although the power of the desired sideband and undesired harmonics can be changed, the total power remains constant. Therefore, this method doesn't require an additional optical amplifier. Since the MZM is operated in linear region and PM tuning helps to minimize the harmonics' power, an additional optical band pass filter can also be avoided.

\section{Theoretical Background}

In this section, we firstly provide the analytical descriptions of series MZM and PM for generating OSSB and OVSB modulation schemes. Then we provide the major optical impairments associated with the general RoF system.

\subsection{Analytical Expressions}

Let $E_{\text {in }}(t)=A_{\mathrm{c}} \exp \left(\mathrm{j} \omega_{\mathrm{c}} t\right)$ be an optical carrier and $\mathrm{M}(t)=\cos \left(\omega_{\mathrm{d}} t\right)$ a modulation electrical signal where $\omega_{\mathrm{c}}$ and $\omega_{\mathrm{d}}$ denote the frequency of the optical carrier and electrical signal, respectively. The general expression for the output electric field from the MZM can be given as [13]

$$
E_{\text {out } 1}(t)=\frac{A_{\mathrm{c}}}{2}\left[\exp \left(\frac{\mathrm{j} \pi M(t)}{V_{\pi}}\right)+\exp \left(\frac{\mathrm{j} \pi V_{\mathrm{b}}}{V_{\pi}}\right)\right]
$$


where $V_{\pi}$ is the half wave voltage of the modulator and $V_{\mathrm{b}}$ is the applied dc bias to the modulator. Next we denote $m=\pi M(t) / V_{\pi}$ the phase modulation index for MZM and $c=\pi V_{\mathrm{b}} / V_{\pi}$ the constant phase shift to the MZM. Now simplifying the above equation yields

$$
\begin{aligned}
& E_{\text {out } 1}(t)= \\
& A_{\mathrm{c}}\left\{\cos (c) \cdot \cos \left[m \cdot \cos \left(\omega_{\mathrm{d}} t\right)\right]-\sin (c) \cdot \sin \left[m \cdot \cos \left(\omega_{\mathrm{d}} t\right)\right]\right\} .
\end{aligned}
$$

Equation (2) can be simplified further using Bessel formulae. Let $p=A_{\mathrm{c}} \cos (c) \cdot \mathrm{J}_{0}(m)$ and $q=A_{\mathrm{c}} \sin (c) \cdot \mathrm{J}_{1}(m)$ for calculation convenience where $\mathrm{J}_{x}, x=0,1 \ldots$ are the coefficients of the Bessel function of the first kind. Taking only the first order sidebands, the above equation will be reduced to

$$
\begin{aligned}
& E_{\text {out } 1}(t)= \\
& E_{\text {in }}(t)\left[p+q \cdot\left(\mathrm{e}^{\mathrm{j} \omega_{\mathrm{d}} t}+\mathrm{e}^{-\mathrm{j} \omega_{\mathrm{d}} t}\right)\right] A_{c}\left\{\mathrm{e}^{\mathrm{j} \omega_{\mathrm{c}} t}\left[p+q \cdot\left(\mathrm{e}^{\mathrm{j} \omega_{\mathrm{d}} t}+\mathrm{e}^{-\mathrm{j} \omega_{\mathrm{d}} t}\right)\right]\right\} .
\end{aligned}
$$

In (3), we neglected the higher order sidebands by assuming the very low power associated with them considering the case of small signal analysis.

Let us suppose that $\sin \left(\omega_{\mathrm{d}} t\right)$ is the PM driving signal. When MZM and PM are connected the field output from $\mathrm{PM}$ can be written as

$$
E_{\text {out } 2}(t)=E_{\text {in } 2}(t) \exp \left[\mathrm{j} \phi \cdot \sin \left(\omega_{\mathrm{d}} t\right)\right]
$$

where $\phi$ is the phase modulation index in radians. The term $E_{\text {in2 }}(t)$ is equal to $E_{\text {out } 1}(t)$. Now simplifying the second term in (4) using Bessel formulae and taking only the first order sidebands yields

$$
E_{\text {out } 2}(t)=E_{\text {in } 2}(t)\left[J_{0}(\phi)+J_{1}(\phi)\left(\mathrm{e}^{\mathrm{j} \omega_{\mathrm{d}} t}-\mathrm{e}^{-\mathrm{j} \omega_{\mathrm{d}} t}\right)\right] .
$$

After simplification and neglecting the higher frequency terms above equation will be reduced to

$$
\begin{aligned}
\frac{E_{\text {out } 2}(t)}{E_{\text {in }}(t)}= & p \cdot J_{0}(\phi)+\left[q \cdot J_{0}(\phi)+p \cdot J_{1}(\phi)\right] \mathrm{e}^{\mathrm{j} \omega_{\mathrm{d}} t}+ \\
& {\left[q \cdot J_{0}(\phi)-p \cdot J_{1}(\phi)\right] \mathrm{e}^{-\mathrm{j} \omega_{\mathrm{d}} t} . }
\end{aligned}
$$

In the above equation, the first term is optical carrier, the second term is USB and the third term is LSB. Upon proper adjustment of the modulator parameters, the condition $q \cdot \mathrm{J}_{0}(\phi)=p \cdot \mathrm{J}_{1}(\phi)$ can be satisfied. Then the above equation results in USB based OSSB scheme. Similarly, for the case of $q \cdot \mathrm{J}_{0}(\phi)=-p \cdot \mathrm{J}_{1}(\phi)$ an LSB based OSSB scheme can be generated. OVSB is the case when the partial suppression of the unwanted sideband in the OSSB scheme that results in unequal sideband intensities. This condition occurs when the above equalities are not met perfectly, which can be described by introducing the new scaling parameter $k$, such that $k= \pm\left[q \cdot \mathrm{J}_{0}(\phi)\right] /\left[p \cdot \mathrm{J}_{1}(\phi)\right]$. The value of $k$ depends on the choice of $p, q$ and $\phi$. It determines the extent of sideband suppression. For maximum suppression its value will be 1 which leads to the OSSB scheme and for partial suppres- sion its value can be considered less than 1 resulting in the OVSB scheme.

\subsection{Transmissions Impairments}

The RoF transmission system suffers from various kinds of noise and distortions both in an optical link and in a wireless link. We describe specifically some of the optical link impairments. In an optical link, thermal noise, shot noise, relative intensity noise (RIN), laser chirp, fiber nonlinearity, CD, polarization mode dispersion (PMD) etc. are present like in any other fiber optic link. The $\mathrm{CD}$ is one of the significant linear impairments in fiber optical communication system whose detail description can be obtained in [14], [15]. Most importantly, CD and nonlinearity associated with modulator and square law photo detector are the major factors for impairments in RoF systems.

The ODSB transmission in a dispersive fiber link leads to different phase shifts between the sidebands. The power of the recovered signal after photo detection can be given as follows [3]

$$
P_{\mathrm{RF}}=\cos ^{2}\left[\pi L c D\left(\frac{f_{\mathrm{RF}}}{f_{0}}\right)^{2}\right]
$$

where $c$ is the speed of light, $D$ is the dispersion parameter, $f_{0}$ is the optical carrier frequency and $f_{\mathrm{RF}}$ is the electrical carrier frequency. If the sidebands experience a total phase difference of $180^{\circ}$ relative to the optical carrier the RF signal will be lost. Depending on the amount of phase shifts due to $\mathrm{CD}$, power fading of the detected RF signal occurs. The received signal shows periodic power variation based on the fiber length due to $\mathrm{CD}$. The first null power point can be calculated as

$$
L=\frac{c}{2 D \lambda^{2} f_{\mathrm{RF}}^{2}}
$$

where $L$ is fiber length and $\lambda$ is the laser wavelength. The first null power points for the $1550 \mathrm{~nm}$ wavelength, $18 \mathrm{ps} /(\mathrm{nm} \mathrm{km})$ dispersion parameter can be calculated for 10,30 and $60 \mathrm{GHz}$ frequencies as $34.68 \mathrm{~km}, 3.85 \mathrm{~km}$ and $0.96 \mathrm{~km}$, respectively. From this calculation it is very clear that $\mathrm{CD}$ is the serious impairment as the RF increases. For $5 \mathrm{G}$ based system to support the bandwidth hungry devices and applications the millimeter wave frequencies are likely to be used due to the congestion of the conventional RF bands. In higher frequency band the chromatic dispersion affects severely. Therefore the CD compensation is a major challenge which limits the system performance. To overcome the $\mathrm{CD}$ effect, $\mathrm{CD}$ tolerant sideband modulation schemes such as OSSB or OCS-DSB have to be used. For higher frequency RoF system, the performance will be largely determined by the extent of the $\mathrm{CD}$ effect reduction.

Due to the cosine transfer characteristic of the MZM, infinite numbers of harmonic products are generated. The harmonic distortion (HD) mainly depends on the used modulation index. When a higher modulation index is used, harmonics' intensities will be increased thereby leading to 
distortion. Each harmonic component experiences different phase change due to $\mathrm{CD}$ in the fiber. The beating of unwanted harmonic components with the first order sideband causes a reduction of the signal strength. Also, the beating of the frequency component can result in new unwanted frequency components. Suppression of these unwanted components can be very difficult. In higher modulation index condition, the intensity of these components will be high leading to serious distortion.

\section{Experimental Validation}

The idea of the proposed method of generating an OVSB scheme was verified by experimental measurements. The experimental arrangement is shown in Fig. 1. In the setup the MZM is a chirpless modulator. The half wave voltage of MZM is $8 \mathrm{~V}$. A continuous wave DFB laser is operated at $1 \mathrm{dBm}$ output power and emission frequency of 193.5 THz. The driving signal for modulators is a sine wave with a frequency of $4 \mathrm{GHz}$. The MZM is biased at the quadrature point. $\mathrm{PM}$ is driven by the $90^{\circ}$ phase shifted version of the same electrical signal as MZM for generating OSSB and OVSB schemes. The used optical spectrum analyzer (OSA) has a resolution of $3 \mathrm{pm}$ which enables visualization of the generated sidebands.

For the MZM dc bias from $-8 \mathrm{~V}$ to $+8 \mathrm{~V}$ and using fixed RF signal driving voltage, two USB based OSSB and two LSB based OSSB schemes and two OCS-DSB schemes were observed. The PM modulation index was varied by changing its driving voltage. The voltage attenuator and amplifiers were utilized for providing the driving voltages. The OVSB scheme can be considered as an unequal intensity of the first order sidebands. For instance, at $1.5 \mathrm{~V}$ of PM driving voltage and $1.9 \mathrm{~V}$ of MZM driving voltage, OVSB scheme with OCSR $=0 \mathrm{~dB}$ was obtained as shown in Fig. 2. Sideband suppression of about $18 \mathrm{~dB}$ was obtained from this setup. It was observed that the electrical drive signals should be at $90^{\circ}$ to each other for the maximum suppression of the undesired sideband.

Some slightly complex methods have been reported for achieving the SNR optimization goal [6], [7], [11]. These methods include DMZM with tunable optical filter, using polarization modulators, optical injection locked laser based technique, etc. In our method we use the OVSB scheme for improving the system performance. The presented OVSB scheme generated from simple series MZM

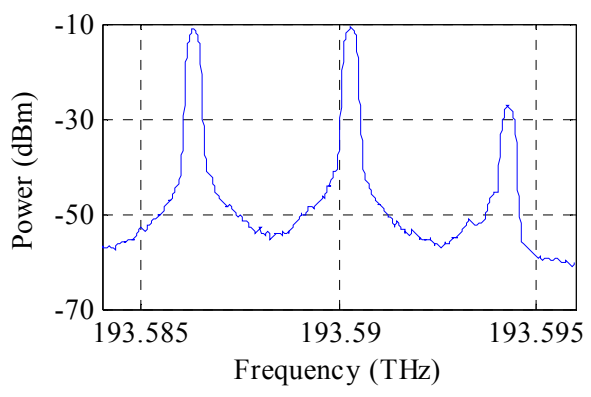

Fig. 2. Measured OVSB scheme. and PM configuration does not require a tunable optical filter to suppress the optical carrier, it also does not require an additional optical amplifier because the total optical power remains constant. With the presented scheme, SNR enhancement can be achieved without the need of tunable optical filter, band pass optical filter and optical amplifier.

\section{Simulation and Discussions}

To compare the performance of the proposed OVSB generation scheme with the OSSB scheme using series MZM and PM configuration, the electrical and optical setup was designed in VPItransmissionMaker simulator along with MATLAB co-simulation. The used setup is the same as in Fig. 1. In the setup, the MZM is a single drive and chirpless modulator. The half wave voltage of MZM is $8 \mathrm{~V}$. The MZM is biased at its quadrature point and the driving signal amplitude is chosen at $0.5 \mathrm{~V}$. The PM is driven by the $90^{\circ}$ phase shifted version of the same electrical signal as MZM. A continuous wave DFB laser is operated at $10 \mathrm{dBm}$ with emission frequency of $193.1 \mathrm{THz}$. The used optical fiber is a standard single mode fiber (SMF). The photo detector type is a positive intrinsic negative $(\mathrm{p}-\mathrm{i}-\mathrm{n})$ diode with a thermal noise parameter of $10^{-12} \mathrm{pA} / \mathrm{Hz}^{1 / 2}$. The fiber dispersion is $18 \mathrm{ps} /(\mathrm{nm} \cdot \mathrm{km})$. The 4QAM signal was used to observe the symbol error rate (SER) performance. The SER was calculated using the Gaussian method.

\subsection{Comparison of OSSB and OVSB Schemes Generated with Series MZM and PM}

The 4QAM, 5 Gbps signal is generated and transmitted over $50 \mathrm{~km}$ of fiber. At PM index of $45^{\circ}$, one of the sidebands is completely suppressed from ODSB resulting in the OSSB scheme. For OVSB the best PM index was calculated based on the SER performance. Optical amplifier and optical filter were not used for all the below discussed simulation results.

Table 1 shows some representative values of the OVSB PM indexes for fiber length of $50 \mathrm{~km}$. Tuning of the $\mathrm{PM}$ index is required for the OVSB scheme generation for each used frequency. The relative sideband power difference of the generated OVSB scheme for each frequency and fiber length will also be different, as for different PM index the harmonics' power relation will change. Thus, the resultant SER of OVSB scheme is due to not only the increased signal power strength but also the proper balance of the harmonics' power distributions. The OVSB scheme enhances the signal power and balances the harmonics power thereby improving the SER.

Figure 3 shows the OSSB and Figure 4 shows the OVSB optical spectra for $20 \mathrm{GHz}$. The OVSB spectrum was obtained with the best SER. The sideband at $+20 \mathrm{GHz}$ in the OSSB scheme is completely suppressed. For the OVSB scheme it is partially suppressed. The OVSB scheme also shows that the second order harmonic power 
at $-40 \mathrm{GHz}$ is more suppressed compared to OSSB scheme. This leads to the reduction of the harmonic distortion. The enhancement of $+20 \mathrm{GHz}$ first order sideband power and reduction of $-40 \mathrm{GHz}$ second order harmonic power leads OVSB scheme to perform better than OSSB scheme. This shows that in series MZM and PM configuration, if proper PM index is selected, the harmonic distortion can be reduced as well as signal strength can be enhanced in comparison with the OSSB scheme.

Figure 5 shows the received signal power for ODSB, OSSB and OVSB modulated signals for various frequency ranges for $50 \mathrm{~km}$ fiber length. As the frequency increases the $\mathrm{CD}$ effect increases leading to a cyclic power variation for ODSB scheme. The CD effect can be minimized with OSSB and OVSB schemes. Thus, the power variation is minimized by using OSSB and OVSB schemes as shown in Fig. 5. The OVSB scheme's signal power strength is higher than other schemes which results in better SER performance.

\begin{tabular}{|c|c|c|c|}
\hline $\begin{array}{c}\text { Frequency } \\
(\mathrm{GHz})\end{array}$ & $\begin{array}{c}\text { OVSB PM } \\
\text { index }\left(^{\circ}\right)\end{array}$ & OSSB SER & OVSB SER \\
\hline 15 & 41 & $10^{-33}$ & $10^{-36}$ \\
\hline 20 & 37 & $10^{-20}$ & $10^{-29}$ \\
\hline 25 & 60 & $10^{-5}$ & $10^{-9}$ \\
\hline 30 & 30 & $10^{-3}$ & $10^{-3}$ \\
\hline
\end{tabular}

Tab. 1. Optimum PM index and improved SER for the OVSB scheme for 4QAM, $5 \mathrm{Gbps}$ system at $50 \mathrm{~km}$ fiber length. The SER were estimated using Gaussian estimation method.

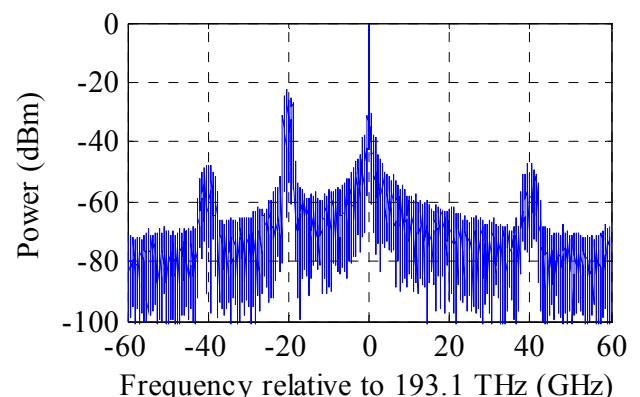

Fig. 3. Optical spectrum for OSSB scheme at $20 \mathrm{GHz}$, the sideband at $20 \mathrm{GHz}$ has been completely suppressed leaving behind $-20 \mathrm{GHz}$ sideband. The sidebands at $\pm 40 \mathrm{GHz}$ are undesired harmonics.

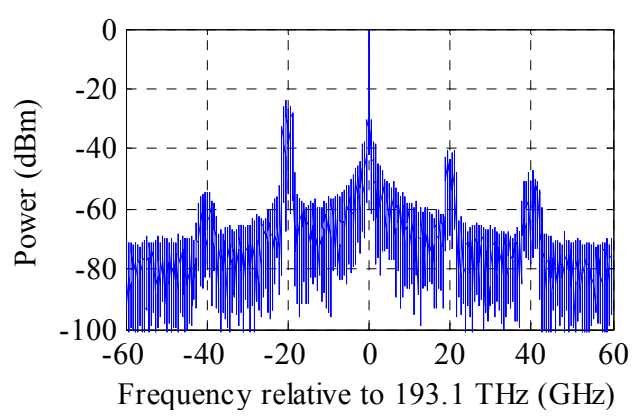

Fig. 4. Optical spectrum for OVSB scheme at $20 \mathrm{GHz}$, the sideband at $+20 \mathrm{GHz}$ has been partially suppressed. The sidebands at $\pm 40 \mathrm{GHz}$ are undesired harmonics in which the USB power level is higher than the LSB. Also the harmonics' power levels are lower than in the case of OSSB scheme.

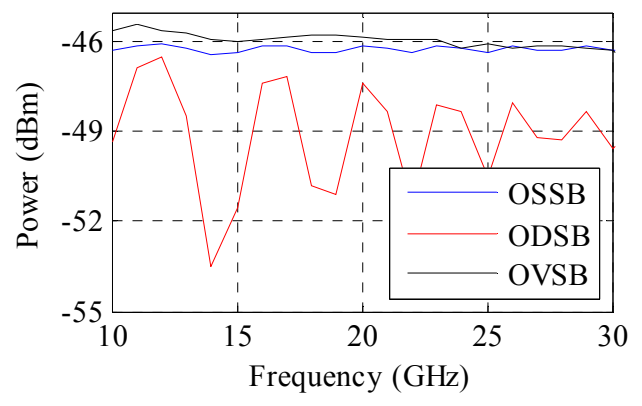

Fig. 5. Received signal power for various sideband modulation schemes for different frequencies at $50 \mathrm{~km}$ fiber length.

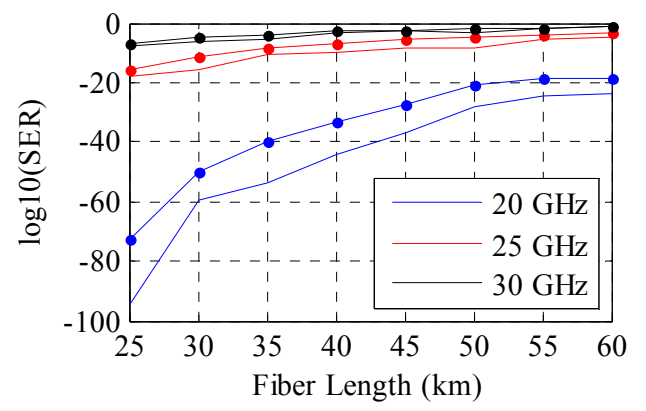

Fig. 6. SER performance of OSSB and OVSB schemes for different frequencies and fiber lengths for $5 \mathrm{Gbps}$, 4QAM modulation format. The curves with (.) are for OSSB scheme and solid plain curves are for OVSB scheme. The SER were estimated using Gaussian estimation method.

The SER performance of the OSSB and OVSB of different frequencies for various fiber lengths is shown in Fig. 6. As frequency and fiber length increase, more CD will be accumulated causing poorer performance. For all cases the OVSB scheme shows superior performance compared to the OSSB scheme. Since the OSSB and OVSB schemes are optical sideband modulation schemes, they are transparent to any electrical modulation format's signal. Any higher order electrical modulation format's signal can be applied for performance comparison. For any kind of electrical modulation format's signal the OVSB scheme shows superior performance compared to the OSSB scheme. To work in higher frequency regime such as in millimeter wave, one needs to employ a fiber amplifier to increase the signal strength along with photonic approaches for higher frequency carrier generation and modulation [16-18].

\subsection{Impact of Harmonic Distortion}

As discussed earlier in the previous section, the nonlinear transfer characteristic of the modulator causes creation of an infinite number of sidebands. Among these, second and third order sidebands are considered significant for harmonic distortion. We studied the behavior of the harmonics' power for MZM and series MZM and PM cases at $20 \mathrm{GHz}$ frequency for various fiber lengths. Figure 7 shows the fundamental $\left(\omega_{\mathrm{d}}\right)$, second $\left(2 \omega_{\mathrm{d}}\right)$, and third $\left(3 \omega_{\mathrm{d}}\right)$ order harmonics' power variations with fiber lengths for single MZM and for series MZM and PM cases. The fiber 


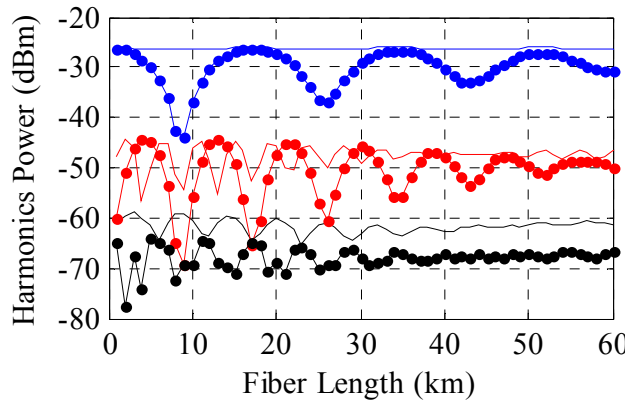

Fig. 7. Harmonic powers. Colors - blue: fundamental, red: second order, black: third order. Lines- with(.): MZM, solid plain: series MZM and PM.

attenuation was not considered for these simulations to observe the harmonics' power variations due to $\mathrm{CD}$.

In Fig. 7 for the single MZM case, first order sideband $\left(\omega_{\mathrm{d}}\right)$ shows its deep power dip at $8.6 \mathrm{~km}$ and $26 \mathrm{~km}$ and so on. When the MZM and PM are connected and the indices are set for OSSB scheme, the $\left(\omega_{\mathrm{d}}\right)$ power variation remains almost constant, showing the $\mathrm{CD}$ compensation. However, the third order sideband $\left(3 \omega_{\mathrm{d}}\right)$ power level is increased slightly, leading to higher third order harmonic distortion than with a single MZM. The cyclic rotation of the second order sideband $\left(2 \omega_{\mathrm{d}}\right)$ for the series MZM and PM case is two times faster than with the single MZM case. As the fiber length increases the levels of the deep power dips (nulls) of $\left(2 \omega_{\mathrm{d}}\right)$ and $\left(3 \omega_{\mathrm{d}}\right)$ are increased showing the increased distortion level.

\section{Conclusions}

In this paper, we presented the series configuration of a MZM and a PM as a flexible approach to generate the optical vestigial sideband modulation scheme (OVSB), which enhances the signal to noise ratio (SNR) compared to the optical single sideband modulation scheme (OSSB). We theoretically verified the proposed generation method and validated through experiment. With the proposed scheme, SNR enhancement can be achieved without the need of tunable optical filter, band pass optical filter and optical amplifier.

We investigated the optimum parameters for OSSB and OVSB schemes and compared the SER performance for 4QAM signal. We showed that the OVSB performance is better than the OSSB scheme in series MZM and PM configuration, if proper PM index is selected. The second and third order distortions were analyzed for both single MZM and series MZM and PM cases. The second order harmonics is a major harmonic distortion contributor in the single MZM case. Third order harmonic has a higher level of power in the proposed series MZM and PM configuration compared to single MZM case. However still second order distortion dominates in the system.

\section{Acknowledgments}

This project has received funding from the European Union's Horizon 2020 research and innovation programme under the Marie Sklodowska-Curie grant agreement No. 642355.

\section{References}

[1] RAPPAPORT, T. S., SUN, S., MAYZUS, R. Millimeter wave mobile communications for $5 \mathrm{G}$ cellular: It will work! IEEE Access, 2013, vol. 1, p. 335-349. DOI: 10.1109/ACCESS.2013.2260813

[2] CHANG, G. K., CHENG, L., XU, M., et al. Integrated fiberwireless access architecture for mobile backhaul and fronthaul in $5 \mathrm{~g}$ wireless data networks. In IEEE Avionics, Fiber-Optics and Photonics Conference (AVFOP). Atlanta (GA, USA), 2014, p. 49 to 50. DOI: 10.1109/AVFOP.2014.6999461

[3] LIM, C., NIRMALATHAS, A., et al. Fiber-wireless networks and subsystems technologies. Journal of Lightwave Technology, 2010, vol. 28, no. 4, p. 390-405. DOI: 10.1109/JLT.2009.2031423

[4] UDVARY, E., BERCELI, T. Combined optical amplification and dispersion compensation in radio over fibre link. IET Optoelectronics, 2010, vol. 4, no. 6, p. 260-266. DOI: 10.1049/ietopt.2009.0061

[5] LIU, C., CHIEN, H. C., FAN, S. H., et al. Enhanced vector signal transmission over double sideband carrier suppressed optical millimetre waves through a small LO feed through. IEEE Photonic Technology Letters, 2012, vol. 24, no. 3, p. 173-175. DOI: 10.1109/LPT.2011.2175719

[6] GAO, Y., WEN, A., PENG, Z. G., et al. Analog photonic link with tunable optical carrier to sideband ratio and balanced detection IEEE Photonics Journal, 2017, vol. 9, no. 2, p. 1-10, 2017. DOI: 10.1109/JPHOT.2017.2667891

[7] HRAIMEL, B., ZHANG, X., PEI, Y., et al., Optical single sideband modulation with tunable optical carrier to sideband ratio in radio over fiber systems. Journal of Lightwave Technology, 2011, vol. 29, no. 5, p. 775-781. DOI: 10.1109/JLT.2011.2108261

[8] ThOMas, V. A., El-HaJjar, M., HANZO, L. Performance improvement and cost reduction techniques for radio over fiber communications. IEEE Communications Surveys \& Tutorials, 2015 , vol. 17 , no. 2, p. 627-670. DOI: 10.1109/COMST.2015.2394911

[9] GAO, Y. S., WEN, A., CHEN, Y., et al. An analog photonic link with compensation of dispersion induced power fading. IEEE Photonic Technology Letters, 2015, vol. 27, no. 12, p. 1301-1304. DOI: 10.1109/LPT.2015.2421271

[10] FANDINO, J. S., MUNOZ, P., DOMENECH, D., CAPMANY, J. A monolithic integrated photonic microwave filter. Nature Photonics, 2017, vol. 11, p. 124-129. DOI: 10.1038/nphoton.2016.233

[11] XIONG, J., WANG, R., PU, T., et al. A novel approach to realizing SSB modulation with optimum carrier to sideband ratio. IEEE Photonics Technology Letters, 2013, vol. 25, no. 12, p. 1114 to 1117. DOI: 10.1109/LPT.2013.2260141

[12] PARAJULI, H. N., UDVARY, E. A vestigial sideband modulation scheme in radio over fiber system using electro-optic modulators. In The 18th International Conference on Transparent Optical Networks (ICTON). Trento (Italy), 2016, p. 1-4. DOI: 10.1109/ICTON.2016.7550477 
[13] THOMAS, V. A., EL-HAJJAR, M., HANZO, L. Millimeter-wave radio over fiber optical upconversion techniques relying on link nonlinearity. IEEE Communications Surveys \& Tutorials, 2016, vol. 18, no. 1, p. 29-53. DOI: 10.1109/COMST.2015.2409154

[14] SAGHAEI, H., HEIDARI, V., EBNALI-HEIDARI, M., et al. A systematic study of linear and nonlinear properties of photonic crystal fibers. Optik - International Journal for Light and Electron Optics, 2016, vol. 127, no. 24, p. 11938-11947. DOI: 10.1016/j.ijleo.2016.09.111

[15] EBNALI HEIDARI M., DEHGHAN F., SAGHAEI, H., et al. Dispersion engineering of photonic fibers by means of fluidic infiltration. Journal of Modern Optics, 2012, vol. 59, no. 16, p. 1384-1390. DOI: $10.1080 / 09500340.2012 .715690$

[16] NOVAK, D., WATERHOUSE, R. B., NIRMALATHAS, A., et al. Radio-over-fiber technologies for emerging wireless systems. IEEE Journal of Quantum Electronics, 2016, vol. 52, no. 1, 11 p. DOI: 10.1109/JQE.2015.2504107

[17] SAFARI, L., BAGHERSALIMI, G., KARAMI, A., et al. On the equalization of an OFDM-based radio-over-fiber system using neural networks. RadioEngineering, 2017, vol. 26, no. 1, p. 162 to 169. DOI: $10.13164 /$ re. 2017.0162

[18] LYSIUK, A., GODZISZEWSKI, K., YASHCHYSHYN, Y., et al. Design and investigation of photonic remote antenna units for bidirectional transmission in the last mile wireless over fiber system. RadioEngineering, 2013, vol. 22, no. 4, p. 1239-1248. ISSN: $1210-2512$

\section{About the Authors...}

Hum Nath PARAJULI is a Marie Curie early stage researcher in the Budapest University of Technology and Economics, Budapest, Hungary. He received his B.Eng. degree from Pokhara University, Nepal in 2008 and joint M.Sc. degree from Osaka University, Japan and Scula Superiore Sant' Anna, Italy in 2012. His current research interests include design of high capacity optical wireless links, millimeter wave communication, digital signal processing for optical communication and design of optoelectronic systems.

Eszter UDVARY received Ph.D. degree in Electrical Engineering from the Budapest University of Technology and Economics (BME), Budapest, Hungary, in 2009. She is currently an Associate Professor at BME, Dept. of Broadband Info-communications and Electromagnetic Theory, where she leads the Optical and Microwave Telecommunication Lab. Dr. Udvary's research interests are in the broad areas of optical communications, include optical and microwave communication systems, Radio over fiber systems, optical and microwave interactions and applications of special electro-optical devices. 